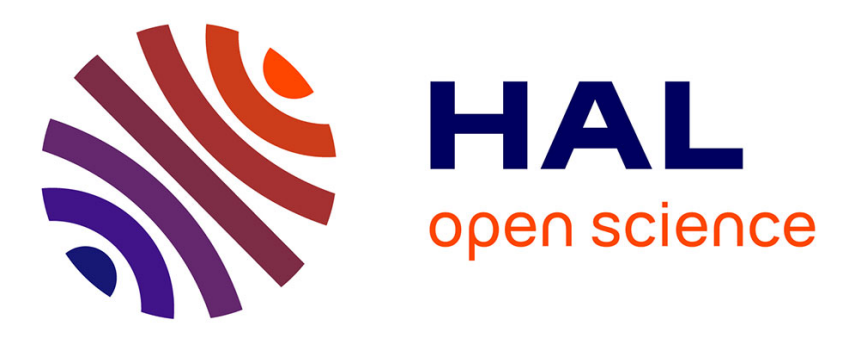

\title{
Circular differential scattering of polarized light by a chiral random medium
}

Jérôme Crassous, Axelle Amon, Jeanne Crassous

\section{To cite this version:}

Jérôme Crassous, Axelle Amon, Jeanne Crassous. Circular differential scattering of polarized light by a chiral random medium. Physical Review A : Atomic, molecular, and optical physics [1990-2015], 2012, 85 (2), pp.023806. 10.1103/PhysRevA.85.023806 . hal-00848202

\section{HAL Id: hal-00848202 https://hal.science/hal-00848202}

Submitted on 20 Aug 2013

HAL is a multi-disciplinary open access archive for the deposit and dissemination of scientific research documents, whether they are published or not. The documents may come from teaching and research institutions in France or abroad, or from public or private research centers.
L'archive ouverte pluridisciplinaire HAL, est destinée au dépôt et à la diffusion de documents scientifiques de niveau recherche, publiés ou non, émanant des établissements d'enseignement et de recherche français ou étrangers, des laboratoires publics ou privés. 


\title{
Circular differential scattering of polarized light by a chiral random medium
}

\author{
Jérôme Crassous* and Axelle Amon \\ Institut de Physique de Rennes, UMR CNRS 6251, Université de Rennes 1, Campus de Beaulieu, F-35042 Rennes Cedex, France \\ Jeanne Crassous \\ Sciences Chimiques de Rennes, UMR CNRS 6226, Université de Rennes 1, Campus de Beaulieu, F-35042 Rennes Cedex, France
}

(Received 22 May 2011; published 7 February 2012)

\begin{abstract}
Differential scattering of left- and right-handed circularly polarized waves is a general property of scattering media lacking space-inversion symmetry. We show in this paper that this scattering difference has remarkable properties in the case of an almost transparent chiral random medium. By tuning the refractive indices of the materials composing the medium, this scattering difference can be varied and even inverted. Within the limit of a dense scattering material, the difference of scattering cross sections between polarizations of opposite handedness can be largely adjusted. These properties are illustrated by measurement of the difference between the transmission of right- and left-handed polarized waves through a chiral Christiansen filter.
\end{abstract}

DOI: 10.1103/PhysRevA.85.023806

PACS number(s): 33.55.+b, 78.20.Ek, 42.25.Dd, 42.25.Fx

\section{INTRODUCTION}

Chirality in a medium affects propagation of vectorial waves. Pasteur first identified that molecular asymmetry in a material causes rotation of the plane of polarization of a linearly polarized wave [1]. This effect may be interpreted as a consequence of the difference between the refractive indices $n_{R}$ and $n_{L}$ of right- and left-handed circularly polarized waves. A consequence of this index difference is illustrated by the separation of a linearly polarized wave into two rays of opposite handedness [2-4], or the difference of scattering properties of an achiral grating embedded in a chiral material [5]. Many fields of research have special interest in the scattering properties of chiral media. For example, development of metamaterials with adjusted dielectric properties has motivated research on circular differential scattering (CDS) by a two-dimensional (2D) chiral grating [6,7]. In biochemistry, where chirality is a major issue, most characterizations use diffusion methods, motivating CDS studies [8]. Moreover, as biological systems are generally turbid, light transport in heterogeneous chiral media has very practical applications. For example, suspension of polystyrene beads in an optically active liquid has been used to mimic the scattering properties of biological tissues $[9,10]$.

From a theoretical point of view, the effect of chirality on the scattering properties of light in a disordered material was first studied by Perrin [11]. For a material made of chiral particles, the scattering matrix is no longer symmetric, and the scattering properties are dependent on the handedness of the waves. As the difference in scattered fields for right- or left-handed incident waves depends on the difference $n_{R}-n_{L}$, which is generally very small, systems exhibiting CDS are considered to be uncommon. Nevertheless, this effect has been put forward to explain distortion in circular dichroism spectra $[8,12]$. CDS has mainly been reported in materials where the chirality of the geometrical structure has a length scale near the optical wavelength. Examples are the scattering of light by helical objects with pitch of micrometric length [13],

\footnotetext{
*jerome.crassous@univ-rennes1.fr
}

cholesteric liquid crystals [14], or chiral objects of millimetric size illuminated by millimetric electromagnetic waves $[15,16]$.

The object of this paper is to show that this difference between the scattering of left and right circularly polarized waves can become arbitrarily large even for a constant, small difference $n_{R}-n_{L}$. The ellipticity of the transmitted wave can then be tuned independently of the optical rotation. That result is counterintuitive, as it seems to be in contradiction with the Kramers-Kronig theorem linking circular dichroism to optical rotation [17]. To demonstrate that effect, we use a chiral Christiansen filter [18], where the difference in circular scattering can be finely controlled by varying the refractive indices of the constituent materials. The circular differential scattering is then shown to strongly depend on the difference between the refractive index $n_{1}$ of the isotropic phase and the average index $n$ of the chiral phase. A theoretical description is given, leading to the prediction of a nontrivial symmetry: exchanging the chiral media with its enantiomer $n_{R} \leftrightarrow n_{L}$ [Fig. 1 (I) $\rightarrow$ (II)] is equivalent to the permutation of the indices of the two phases $n_{1} \leftrightarrow n$ [Fig. 1 (I) $\rightarrow$ (III)]. This effect is evidenced experimentally.

\section{EXPERIMENT}

We use as the scattering medium a dispersion of dielectric spherical particles in an optically active fluid. The particles are polymethyl methacrylate (PMMA) beads (Atochem, diameter $d=100-250 \mu \mathrm{m})$. The optically active fluid is an $85 \%: 15 \%$ mixture of limonene $(R, S$, or a mixture of $R$ and $S$; SigmaAldrich) with high-refractive-index microscopy oil (Santolight $n_{\text {oil }}=1.62$ ) in order to reach $n \simeq n_{1}$. The optical activity of the fluid is measured by the optical rotation angle through an $L=20 \mathrm{~mm}$ cell. The PMMA particles are poured into the liquid, and the cell is gently tapped. The resultant solid fraction is $\sim 0.60 . n_{1}-n$ is varied continuously by controlling the temperature of the composite material. The variation of the refractive index of PMMA with temperature is obtained from [19] $d n_{1} / d T=-1.20 \times 10^{-4} \mathrm{~K}^{-1}$. The variation of refractive index of the limonene-oil mixture is obtained from the variations of the constituents [20]: $d n / d T=$ $-4.28 \times 10^{-4} \mathrm{~K}^{-1}$. 




(b)

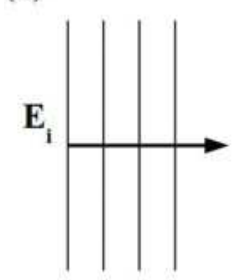



(III)

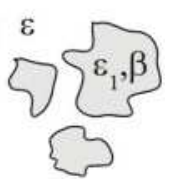

FIG. 1. (Color online) (a) Middle: a chiral material (I) differentially transmits right- and left-handed circular waves. Left (II): Symmetry imposes that the difference in transmission of right- and left-handed circular waves is reversed for the mirror material. Right (III): Near index matching, the permutation of refractive indices also reverses the transmission of right- and left-handed circular waves. (b) (I): A chiral medium with dielectric constant $\epsilon$ and chiral parameter $\beta$ [see (1)] dispersed in a medium $\epsilon_{1}$ scatters an incident wave $\mathbf{E}_{i}$. (II): Same as (I) but the chiral medium has been changed to its enantiomer. (III): Same as (I) but the refractive indices have been permuted.

A schematic drawing of the experimental setup is shown in Fig. 2(a). The cell temperature $T$ is controlled to a few millikelvin precision, and the temperature is increased from 34 to $42{ }^{\circ} \mathrm{C}$ at a rate of $0.15^{\circ} \mathrm{C} / \mathrm{min}$. The cell is illuminated with a low-power He-Ne laser operating at $\lambda=633 \mathrm{~nm}$, and the transmitted light is collected by a $15 \mathrm{~mm}^{2}$ square photodiode (PD), centered $50 \mathrm{~cm}$ after the exit of the cell. We first discuss the transmission $I / I_{0}$ as a function of $n_{1}-n$, for a linearly polarized incident wave [Fig. 2(b), dotted line]. The value $n_{1}-$ $n$ is set to 0 at the maximum of the transmission curve. The bellshaped curve is typical of transmission through Christiansen filters. Near index matching, the medium is almost transparent, and the transmission gradually decreases with the quality of the index matching. The finite width of the transmission curve and the imperfect transmission at index matching are due to fluctuations of the refractive indices around their mean values. Although models for transmission of Christiansen filters are still unclear [18], we can use [21] $I / I_{0}=\exp \left\{-A\left[\left(n_{1}-n\right)^{2}+\right.\right.$ $\left.\left.\sigma^{2}\right]\right\}$ with $A$ a constant dependent on $L, \lambda, d$, and the solid fraction, and where $\sigma$ is the variance of the solid refractive index. From the experimental transmission for $n_{1} \simeq n$ [see the inset of Fig. 2(b)], we deduce $\sigma \simeq 2.8 \times 10^{-4}$.

The differential scattering between circular polarizations is obtained by modulation of the incident polarization with a photoElastic modulator (PEM). The imposed phase modulation is $42 \mathrm{kHz}$ with a delay between the fast and slow axes of the PEM of $\lambda / 4$. The modulation of the transmitted
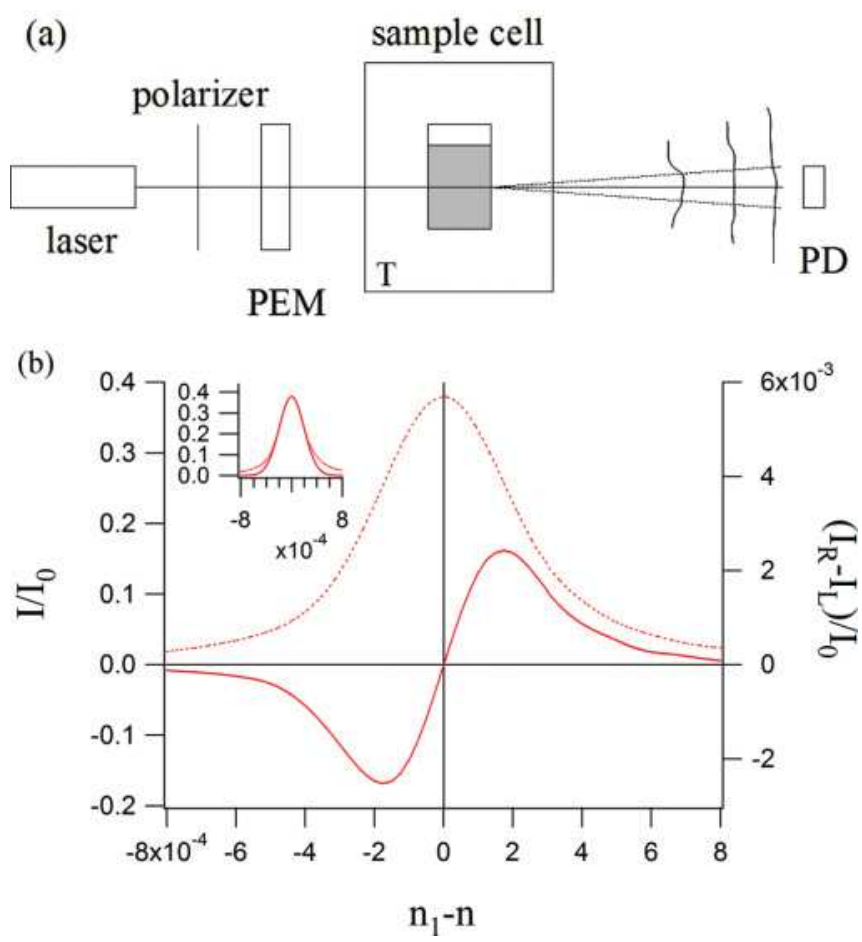

FIG. 2. (Color online) (a) Schematic of the experiment (see text for details). (b) Dotted line, transmission of the cell as a function of the refractive index difference. Solid line, difference of transmission between left and right circular polarization. Inset: Dotted line, transmission. Solid line, fit with $\exp \left\{-A\left[\left(n_{1}-n\right)^{2}+\sigma^{2}\right]\right\}$.

intensity is measured with a lock-in amplifier (Stanford Research SR830). The difference of transmission between right and left circular polarization is plotted as a function of the index matching $n_{1}-n$ [Fig. 2(b), solid line]. We clearly see that the difference of transmission between opposite circular polarizations depends not only on the presence of asymmetry but also on the contrast of the refractive indices $n_{1}-n$.

\section{THEORY}

The order of the chiral contribution to the scattered wave can be estimated. The constitutive relations for an optically active material are [22]

$$
\mathbf{D}=\epsilon \mathbf{E}+\gamma \epsilon \nabla \times \mathbf{E}, \quad \mathbf{B}=\mu \mathbf{H}+\beta \mu \nabla \times \mathbf{H},
$$

where $\gamma$ and $\beta$ are parameters responsible for the optical activity. For simplicity, we restrict ourselves to materials where $\gamma=\beta$ and $\mu=\mu_{0}$, and without any circular dichroism [23]. The refractive indices for right $n_{R}$ and left $n_{L}$ monochromatic circularly polarized waves satisfy the relations $\left(\frac{1}{n_{R}}+\frac{1}{n_{L}}\right)^{-1}=$ $\frac{1}{2} \frac{\omega}{k} \sqrt{\epsilon \mu_{0}}$ and $\frac{1}{n_{R}}-\frac{1}{n_{L}}=2 \beta k$, where $k$ is the wave vector in vacuum, and $\omega$ is the wave frequency. When such a material is dispersed in a medium of dielectric constant $\epsilon_{1}$, Eq. (1) may be written as

$$
\mathbf{D}=\epsilon_{1} \mathbf{E}+\mathbf{P}_{1}, \quad \mathbf{B}=\mu_{0}(\mathbf{H}+\mathbf{M})
$$

with $\mathbf{P}_{1}=\left(\epsilon-\epsilon_{1}\right) \mathbf{E}+\beta \epsilon \nabla \times \mathbf{E}$ and $\mathbf{M}=\beta \nabla \times \mathbf{H}$, which can be respectively considered as the polarization and magnetization of the chiral medium with reference to the isotropic phase. $\mathbf{P}_{1}$ and $\mathbf{M}$ may therefore be viewed as the 
source of the scattered field created by a plane wave propagating with a wave vector $k_{1}=n_{1} k$, with $n_{1}^{2}=\epsilon_{1} / \epsilon_{0}$. The nonchiral contribution to the scattered field scales as $\left(\epsilon-\epsilon_{1}\right) E$ and the chiral one as $\beta \epsilon k_{1} E$. The ratio of those contributions varies then as $\sim \beta k_{1} \epsilon /\left(\epsilon-\epsilon_{1}\right) \approx\left(n_{R}-n_{L}\right) / 2\left(n^{2}-n_{1}^{2}\right)$. If the isotropic medium is a vacuum, $n_{1}=1$, and, as $\mid n_{R}-$ $n_{L} \mid \ll n$, the contribution of chirality to the scattered field stays very small. In contrast, for a dispersion approaching phase matching, $\epsilon_{1} \rightarrow \epsilon$, and the effect of chirality on the scattered field becomes preponderant.

For analysis of the differential transmission between the left- and right-handed circular polarization, we need to calculate the variation of the amplitude of the scattered wave as a function of $\left(\epsilon-\epsilon_{1}\right)$ and of $\beta$. Let us consider an incident wave $\mathbf{E}_{i}(\mathbf{r}, t)=\operatorname{Re}\left[\tilde{\mathbf{E}}_{i}(\mathbf{r}, \omega) \exp (-j \omega t)\right]$, with a similar dependence for $\mathbf{H}_{i}(\mathbf{r}, t)$. The tilde indicates a complex quantity, and we will ignore the $\omega$ dependencies in the following analysis. The incident wave propagates into a homogeneous medium with dielectric constant $\epsilon_{1}$ and magnetic constant $\mu_{0}$. Then a perturbation medium characterized by polarization $\tilde{\mathbf{P}}_{1}(\mathbf{r})=$ $\left(\epsilon-\epsilon_{1}\right) \tilde{\mathbf{E}}(\mathbf{r})+\beta \epsilon \nabla \times \tilde{\mathbf{E}}(\mathbf{r})$ and magnetization $\tilde{\mathbf{M}}(\mathbf{r})=\beta \nabla \times$ $\tilde{\mathbf{H}}(\mathbf{r})$ is added to the homogeneous medium. The field $\tilde{\mathbf{E}}(\mathbf{r})$ in the presence of the perturbation medium may be written as $\tilde{\mathbf{E}}(\mathbf{r})=\tilde{\mathbf{E}}_{i}(\mathbf{r})+\tilde{\mathbf{E}}_{s}(\mathbf{r})$, where the scattered electric field is [24]

$$
\tilde{\mathbf{E}}_{s}(\mathbf{r})=\frac{1}{\epsilon_{1}}\left[\nabla \times \nabla \times \tilde{\boldsymbol{\Pi}}_{e}(\mathbf{r})+j \frac{n_{1} k_{1}}{c} \nabla \times \tilde{\boldsymbol{\Pi}}_{m}(\mathbf{r})-\tilde{\mathbf{P}}_{1}(\mathbf{r})\right]
$$

with $\tilde{\boldsymbol{\Pi}}_{e}(\mathbf{r})=\frac{1}{4 \pi} \int_{V} \tilde{\mathbf{P}}_{1}\left(\mathbf{r}^{\prime}\right) \frac{\exp \left(j k\left|\mathbf{r}-\mathbf{r}^{\prime}\right|\right)}{\left|\mathbf{r}-\mathbf{r}^{\prime}\right|} d \mathbf{r}^{\prime}$ the electric Hertz vector, $\tilde{\boldsymbol{\Pi}}_{m}(\mathbf{r})=\frac{1}{4 \pi} \int_{V} \tilde{\mathbf{M}}\left(\mathbf{r}^{\prime}\right) \frac{\exp \left(j k\left|\mathbf{r}-\mathbf{r}^{\prime}\right|\right)}{\left|\mathbf{r}-\mathbf{r}^{\prime}\right|} d \mathbf{r}^{\prime}$ the magnetic Hertz vector, and $V$ the volume of the perturbation medium. At a point $\mathbf{r}=r \mathbf{s}$ far from the scattering medium, $\tilde{\boldsymbol{\Pi}}_{e}(\mathbf{r})$ and $\tilde{\boldsymbol{\Pi}}_{m}(\mathbf{r})$ vary as $\frac{\exp \left(j k_{1} r\right)}{r}$, and at any point outside the scattering medium $\tilde{\mathbf{P}}_{1}(\mathbf{r})=\mathbf{0}$ and $\tilde{\mathbf{M}}(\mathbf{r})=\mathbf{0}$. The scattered field may then be written as

$$
\tilde{\mathbf{E}}_{s}(r \mathbf{s}) \sim \tilde{\mathbf{A}}(\mathbf{s}) \frac{\exp \left(j k_{1} r\right)}{r}, \quad \tilde{\mathbf{H}}_{s}(r \mathbf{s}) \sim \frac{n_{1}}{\mu_{0} c} \mathbf{s} \times \tilde{\mathbf{E}}_{s}(r \mathbf{s})
$$

with

$$
\tilde{\mathbf{A}}(\mathbf{s})=-k^{2}\left(\mathbf{s} \times\left[\mathbf{s} \times \hat{\mathbf{P}}_{1}\left(k_{1} \mathbf{s}\right)\right]+\frac{n_{1}}{c} \mathbf{s} \times \hat{\mathbf{M}}\left(k_{1} \mathbf{s}\right)\right),
$$

where $\quad \hat{\mathbf{P}}_{1}(\mathbf{k})=\int_{V} \tilde{\mathbf{P}}_{1}\left(\mathbf{r}^{\prime}\right) \exp \left(-j \mathbf{k} \cdot \mathbf{r}^{\prime}\right) d \mathbf{r}^{\prime} \quad$ and $\quad \hat{\mathbf{M}}(\mathbf{k})=$ $\int_{V} \tilde{\mathbf{M}}\left(\mathbf{r}^{\prime}\right) \exp \left(-j \mathbf{k} \cdot \mathbf{r}^{\prime}\right) d \mathbf{r}^{\prime}$. For weakly scattering media, $E_{s} \ll E_{i}$, and $\tilde{\mathbf{P}}_{1}$ and $\tilde{\mathbf{M}}$ may be evaluated for the incident field. For a plane circular wave, $\tilde{\mathbf{E}}_{i}(\mathbf{r})=\tilde{\mathbf{E}}_{i} \exp \left(j k_{1} \mathbf{s}_{0} \cdot \mathbf{r}\right)$, where $\mathbf{s}_{0}$ is the incident direction. For scattering directions $\mathbf{s} \approx \mathbf{s}_{0}$, we then obtain

$$
\tilde{\mathbf{A}}(\mathbf{s}) \sim \mathbf{s} \times\left\{\mathbf{s}_{0} \times\left[\left(\epsilon-\epsilon_{1}\right) \pm k_{1} \beta\left(\epsilon+\epsilon_{1}\right)\right] \hat{\Theta}\left(\mathbf{s}_{0}, \mathbf{s}\right) \tilde{\mathbf{E}}_{i}\right\}
$$

with $\hat{\Theta}\left(\mathbf{s}_{0}, \mathbf{s}\right)=\int_{V} \Theta(\mathbf{r}) \exp \left[-j k_{1}\left(\mathbf{s}-\mathbf{s}_{0}\right) \cdot \mathbf{r}^{\prime}\right] d \mathbf{r}^{\prime}, \quad$ where $\Theta(\mathbf{r})=0$ if $\mathbf{r}$ is in the solvent and 1 otherwise; the $-(+)$ sign stands for right-handed (left-handed) circular waves. The circular differential scattering is found in the first Born approximation and not in the second Born approximation as in [25] because we use nonisotropic polarization. The total scattered intensity is obtained by integration of $\tilde{\mathbf{A}}(\mathbf{s}) \cdot \tilde{\mathbf{A}}^{*}(\mathbf{s})$ over all scattering directions and is $I_{s} \propto\left[\left(\epsilon-\epsilon_{1}\right) \pm \beta k_{1}\left(\epsilon+\epsilon_{1}\right)\right]^{2}$. With $\delta n=n-n_{1}$ and $\Delta n=n_{R}-n_{L}$, we obtain for $\left|n_{R}-n_{L}\right| \ll n$

$$
I_{s} \propto\left[\delta n \pm \frac{\Delta n}{2}\right]^{2}
$$

The transmitted intensity being $I_{0}-I_{S}$, the difference between the transmission $I_{R}$ of right-handed and $I_{L}$ of left-handed circularly polarized waves is then in our approximation $I_{R}-I_{L} \propto \Delta n \delta n$. More generally, considering departure from the weakly scattering case, we calculate that $I_{s}$ remains a function of $\left(\delta n \pm \frac{\Delta n}{2}\right)$ at successive orders, so that

$$
I_{R}-I_{L}=\Delta n \frac{d I}{d \delta n}
$$

where $I=\left(I_{R}+I_{L}\right) / 2$.

\section{DISCUSSION}

We plot in Fig. 3(a) the experimental difference $\left[I_{R}-\right.$ $\left.I_{L}\right] / I_{0}$ as a function of the derivative of $I / I_{0}$ with respect to $\delta n$. Each line corresponds to a different mixture of $R$ and $S$ limonene. As expected, the scattering difference between right- and left-handed circular polarization varies proportionally to the derivative of the transmitted intensity. The value


FIG. 3. (Color online) (a) Experimental difference of transmission between left and right circular waves as a function of the derivative of the total transmitted light with respect to the difference of refractive indices $\delta n$. Solutions are oil with limonene $(R)$ and $(S)$ and mixes $(M 1)$ and $(M 2)$ of $(R)$ and $(S)$. (b) Measured slopes from (a) plotted as a function of $\Delta n$; the solid line corresponds to Eq. (8). 
of $\Delta n$ may be obtained from the slope $\left[I_{R}-I_{L}\right] /(d I / d \delta n)$. Figure 3(b) shows a plot of the deduced slopes as a function of $\Delta n$, measured independently from the rotation of the plane of polarization of light propagation through the liquid alone. We observe that Eq. (8) holds even far from the weakly scattering region, showing that our generalization beyond the weak-scattering limit is convincing.

The fluctuation $\sigma$ of the refractive index of the beads diminishes the intensity of the transmitted light. Remarkably, the fluctuations do not modify the relation (8) even if $\Delta n \ll \sigma$. These fluctuations may be taken into account by considering $\epsilon(\mathbf{r})=\bar{\epsilon}+\delta \epsilon(\mathbf{r})=\bar{\epsilon}+\delta \epsilon \varphi(\mathbf{r})$, where $\bar{\epsilon}$ is the average of $\epsilon(\mathbf{r}), \delta \epsilon(\mathbf{r})$ is the fluctuation, $\delta \epsilon$ is the amplitude of the fluctuation, and $\varphi(\mathbf{r})$ is a function of order unity. Integration of $\tilde{\mathbf{A}}(\mathbf{s}) \cdot \tilde{\mathbf{A}}^{*}(\mathbf{s})$ over all the scattering directions shows that to the highest order $I_{s} \propto\left[\left(\epsilon-\epsilon_{1}\right) \pm \beta k_{1}\left(\epsilon+\epsilon_{1}\right)\right]^{2}+\alpha[\delta \epsilon]^{2}$ with $\alpha=\int_{\mathbf{s}} \hat{\varphi}(\mathbf{s}) \hat{\varphi}^{*}(\mathbf{s}) d \mathbf{s} / \int_{\mathbf{s}} \hat{\theta}(\mathbf{s}) \hat{\theta}^{*}(\mathbf{s}) d \mathbf{s}$. So the fluctuations of $\epsilon(\mathbf{r})$ cancel out in the difference $I_{R}-I_{L}$ and in the derivative $d I / d \delta n$, and Eq. (8) still holds. Still, those fluctuations are responsible for the limitation of CDS by preventing divergence of $d I / d \delta n$ near phase matching.

Finally, Eq. (7) shows that the same value of $I_{R}-I_{L}$ can be obtained by simultaneously exchanging the material with its mirror image ( $\Delta n \rightarrow-\Delta n)$ and by changing $\epsilon$ to $\epsilon_{1}(\delta n \rightarrow$ $-\delta n)$. This may be simply understood. Let $\mathbf{E}_{s}^{\text {(II) }}$ and $\mathbf{E}_{s}^{(\text {III) }}$ be the fields scattered in the two experiments (II) and (III) depicted in Fig. 1(b). If we now consider the superposition of the two sources of radiation, the medium is homogeneous, and the total scattered field is null. Linearity of the Maxwell equations and of the constitutive equations (1) gives $\mathbf{E}_{s}^{(\mathrm{II})}+\mathbf{E}_{s}^{(\mathrm{III})}=0$. Thus, the scattered intensities in the two experiments are the same. That effect is indeed observed experimentally, as shown by the measurements for enantiomeric solutions on Fig. 3(a) and the symmetric form of the curves of Fig. 2(b). That invariance can be seen as a macroscopic analog of the $C P$ symmetry [26]. A chiral medium lacks parity symmetry. In order to obtain invariance, charge conjugation should also be performed if time-inversion invariance is assumed $[26,27]$. Consider first the exchange of indices between two achiral media: i.e., changing the index of the inclusions from $\epsilon_{1}+\delta \epsilon$ to $\epsilon_{1}-\delta \epsilon$ while keeping the index of the surrounding medium constant at $\epsilon_{1}$. The effect of the transformation will be to change the sign of the polarization and consequently the sign of the scattered electric field. Such a transformation then gives the same result as a charge conjugation. Considering now chiral inclusions, the combination of the two transformations of charge conjugation and parity on the medium will result in an identical response to an excitation.

\section{CONCLUSIONS}

To conclude we have shown that circular differential scattering can be tuned in a weakly scattering material having a small constant value of $n_{R}-n_{L}$ : it can be canceled, inverted, or adjusted in magnitude. Remarkable symmetry properties have been evidenced and justified.

\section{ACKNOWLEDGMENTS}

The authors thank P. Snabre for discussions and for the PMMA beads, G. Loas for help with the optical modulator measurement, and the team "Photonique et Laser" of Institut de Physique de Rennes for the loan of the PEM.
[1] L. Pasteur, C. R. Hebd. Séances Acad. Sci. 26, 535 (1848).

[2] A. Fresnel, Ann. Chim. Phys. 28, 147 (1825); in Oeuvres Complètes d'Augustin Fresnel, edited by H. D. Sénarmont, E. Verdet, and L. Fresnel (Paris, Imprimerie Impériale, 1866), Vol. 1.

[3] E. v. Fleischl, Sitzungsber. K. Akad. Wiss. (Math.-Nat.) 90, 478 (1884).

[4] A. Ghosh and P. Fischer, Phys. Rev. Lett. 97, 173002 (2006).

[5] A. Ghosh, F. M. Fazal, and P. Fischer, Opt. Lett. 32, 1836 (2007).

[6] E. Plum, X. X. Liu, V. A. Fedotov, Y. Chen, D. P. Tsai, and N. I. Zheludev, Phys. Rev. Lett. 102, 113902 (2009).

[7] V. A. Fedotov, P. L. Mladyonov, S. L. Prosvirnin, A. V. Rogacheva, Y. Chen, and N. I. Zheludev, Phys. Rev. Lett. 97, 167401 (2006).

[8] D. W. Urry and J. Krivacic, Proc. Natl. Acad. Sci. USA 65, 845 (1970).

[9] M. Kohl, M. Cope, M. Essenpreis, and D. Böcker, Opt. Lett. 19, 2170 (1994).

[10] K. C. Hadley and I. A. Vitkin, J. Biomed. Opt. 7, 291 (2002).

[11] F. Perrin, J. Chem. Phys. 10, 415 (1942).

[12] C. Bustamante, I. Tinoco, and M. F. Maestre, Proc. Natl. Acad. Sci. USA 80, 3568 (1983).

[13] M. F. Maestre, C. Bustamante, T. L. Hayes, J. A. Subirana, and I. Tinoco Jr., Nature (London) 298, 773 (1982).
[14] C. J. Bustamante, K. S. Wells, D. Keller, B. Samori, M. F. Maestre, and I. Tinoco Jr., Mol. Cryst. Liq. Cryst. 111, 79 (1984).

[15] R. D. Haracz, L. D. Cohen, A. Cohen, and R. T. Wang, Appl. Opt. 26, 4632 (1987).

[16] I. Tinoco and M. P. Freeman, J. Phys. Chem. 61, 1196 (1957).

[17] J. A. Schellman, Chem. Rev. 75, 323 (1975).

[18] Carlos R. Hense, Glastech. Ber. 60, No. 3 (1987); 60, No. 4 (1987).

[19] J. M. Cariou, J. Dugas, L. Martin, and P. Michel, Appl. Opt. 25, 334 (1986).

[20] For microscope oil, $d n / d T=-4.2 \times 10^{-4} \mathrm{~K}^{-1}$, Santolight SL5262 data sheet; for limonene, $d n / d T=-4.29 \times 10^{-4} \mathrm{~K}^{-1}$, Landolt-Börstein, doi: 10.1007/978-3-540-75291-2_308.

[21] Equation (1) in [18], from V. I. Shelyubskii, Fiz. Metody Issled. Neorg. Mater. 19, 268 (1976).

[22] E. U. Condon, Rev. Mod. Phys. 9, 432 (1937).

[23] L. D. Barron, Molecular Light Scattering and Optical Activity, 2nd rev. ed. (Cambridge University Press, Cambridge, 2004).

[24] M. Born and E. Wolf, Principles of Optics, 7th ed. (Cambridge University Press, Cambridge, 2003).

[25] C. Bustamante, M. F. Maestre, D. Keller, and I. Tinoco, J. Chem. Phys. 80, 4817 (1984).

[26] J. F. Nieves and P. B. Pal, Am. J. Phys. 62, 207 (1994).

[27] M. Avalos, R. Babiano, P. Cintas, J. L. Jiménez, J. C. Palacios, and L. D. Barron, Chem. Rev. 98, 2391 (1998). 